\title{
Ultra-High Resolution Electron Tomography for Materials Science: a Roadmap
}

\author{
K.J. Batenburg, ${ }^{*}, * *$ S. Bals, ${ }^{* *}$, S. Van Aert, ${ }^{* *}$ T. Roelandts, ${ }^{* *}$ and J. Sijbers, ${ }^{* *}$ \\ * Centrum Wiskunde \& Informatica, P.O. Box 94079, NL-1090 GB Amsterdam, The Netherlands \\ ** Vision Lab, University of Antwerp, Belgium, B-2610 \\ *** Electron Microscopy for Materials Research (EMAT), University of Antwerp, Belgium, B-2020
}

3D imaging techniques for electron microscopy, such as electron tomography [1] and depth sectioning [2,3], are key components in modern materials science research. Observing the structure of a material at various scales allows us to understand its properties and improve its design.

Recently, significant advances have been made in the resolution that can be obtained by such techniques, to the extent that the 3D location of individual atoms, or even the combined assembly of atoms in a nanoparticle [4], can be determined from a series of high resolution EM images. These advances are not only driven by improved microscope resolution, but also by applying more advanced computational procedures to transform the measured EM images into a 3D reconstruction.

As with all experimental techniques, the quality of the final result is limited by the weakest link in the imaging workflow. To fully exploit the capabilities of modern microscopes for high resolution $3 \mathrm{D}$ imaging of a broad range of nanomaterials, it is crucial that the computational model used to reconstruct the 3D image corresponds accurately with the underlying physics. Also, the EM images by themselves often do not contain sufficient information to allow for a well-determined 3D reconstruction: several 3D images can be consistent with the same measured dataset. The key to solving this problem is to incorporate a certain amount of prior knowledge about the material, which can often be obtained from literature, or by experiments with complementary imaging techniques.

In this talk, I will discuss recent results in high resolution 3D imaging of nanomaterials based on HAADF STEM images (Fig. 1), and sketch a roadmap for the years ahead. Significant improvements in the 3D imaging capability can be accomplished by a concerted research effort, covering the entire imaging pipeline, with a focus on:

a. Highly stable experimental conditions and fully automated image acquisition.

b. Accurate and fast simulation of the image formation process, based on a concise model of the underlying physics.

c. Computational Modeling of available prior knowledge of the material (e.g., atomic species, particle morphology, crystal lattice parameters).

d. Reconstruction algorithms that employ the models from (b) and (c) to compute a 3D image that maximizes consistency with the measured EM images, while adhering to the available prior knowledge.

Starting from concrete research questions in materials science, related to the 3D atomic structure of interfaces and core-shell particles [5], challenges and obstacles will be discussed for each of these four aspects of the imaging pipeline. 


\section{References}

[1] P.A. Midgley and R.E. Dunin-Borkowski, Nature Materials, 8 (2009) 271

[2] K. Van Benthem et al., Ultramicroscopy 106 (2006) 1062

[3] A.Y. Borisevich et al., Proc. Natl. Acad. Sci. 103 (2006) 3044

[4] S. Van Aert et al., Nature (to appear, doi: 10.1038/nature09741)

[5] V. Petkov et al., Phyiscal Review B 81 (2010) 241304

[6] This research was supported by the Fund for Scientific Research, Flanders (FWO).

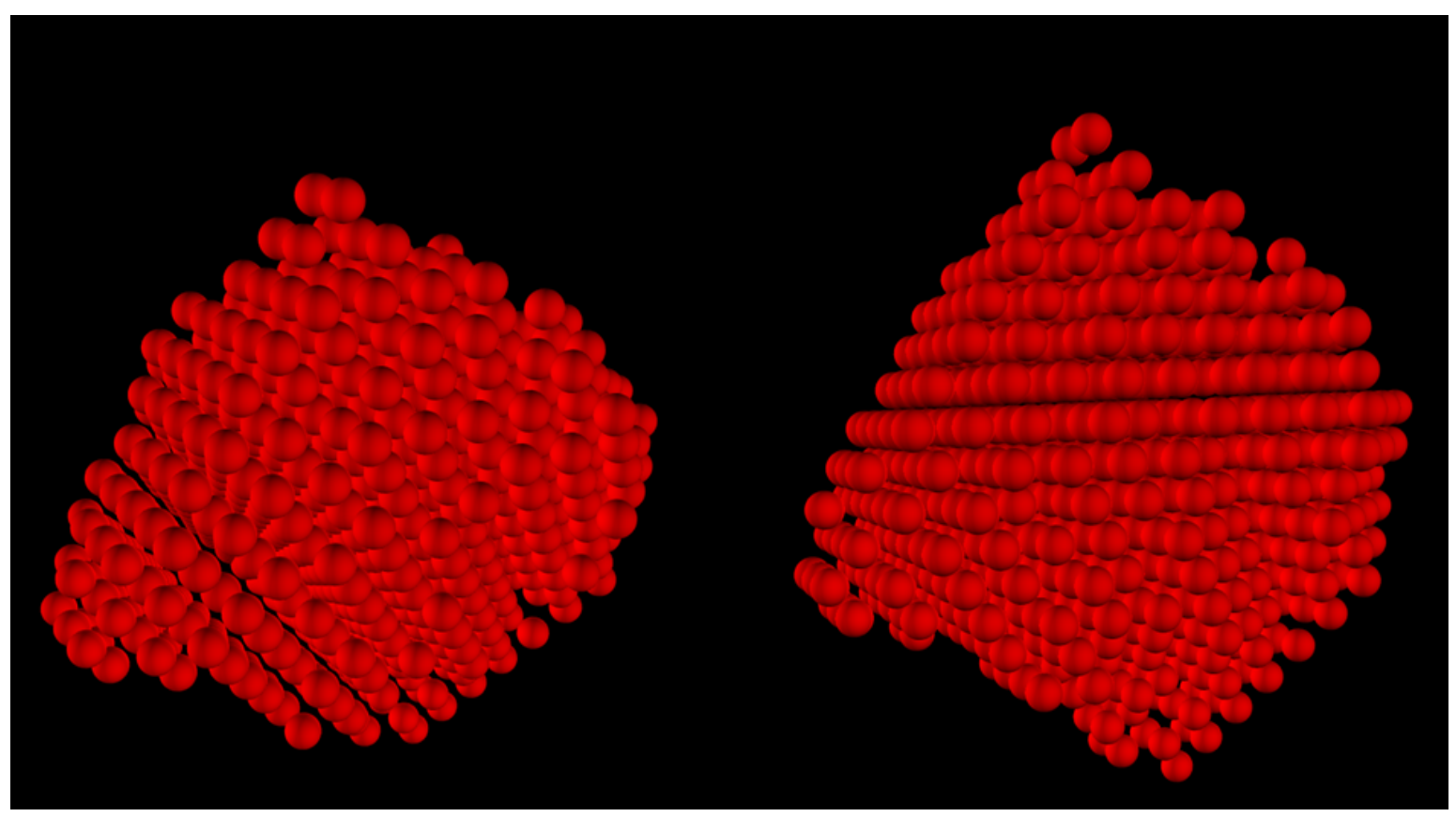

FIG. 1. Two different views of a Ag nanoparticle that was reconstructed from two HAADF STEM projection images by combining parameter estimation techniques with discrete tomography [4]. 